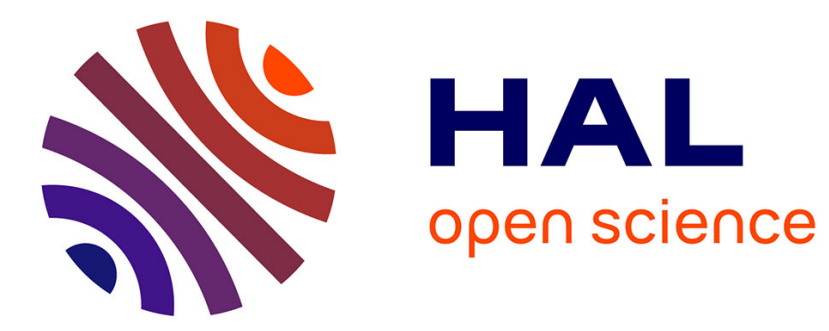

\title{
Automatic Extraction of Developer Expertise
}

Cédric Teyton, Marc Palyart, Jean-Rémy Falleri, Floréal Morandat, Xavier

Blanc

\section{To cite this version:}

Cédric Teyton, Marc Palyart, Jean-Rémy Falleri, Floréal Morandat, Xavier Blanc. Automatic Extraction of Developer Expertise. 2014. hal-00965074

\section{HAL Id: hal-00965074 \\ https://hal.science/hal-00965074}

Preprint submitted on 25 Mar 2014

HAL is a multi-disciplinary open access archive for the deposit and dissemination of scientific research documents, whether they are published or not. The documents may come from teaching and research institutions in France or abroad, or from public or private research centers.
L'archive ouverte pluridisciplinaire HAL, est destinée au dépôt et à la diffusion de documents scientifiques de niveau recherche, publiés ou non, émanant des établissements d'enseignement et de recherche français ou étrangers, des laboratoires publics ou privés. 


\section{Automatic Extraction of Developer Expertise}

\author{
Cédric Teyton \\ Univ. Bordeaux, LaBRI, UMR \\ 5800 \\ F-33400 Talence, France \\ cteyton@labri.fr
}

\author{
Marc Palyart \\ Univ. Bordeaux, LaBRI, UMR \\ 5800 \\ F-33400 Talence, France \\ mpalyart@labri.fr
}

\author{
Jean-Rémy Falleri \\ Univ. Bordeaux, LaBRI, UMR \\ 5800 \\ F-33400 Talence, France \\ falleri@labri.fr
}

\author{
Floréal Morandat \\ Univ. Bordeaux, LaBRI, UMR \\ 5800 \\ F-33400 Talence, France \\ fmorandat@labri.fr
}

\author{
Xavier Blanc \\ Univ. Bordeaux, LaBRI, UMR \\ 5800 \\ F-33400 Talence, France \\ xblanc@labri.fr
}

\begin{abstract}
Context: Expert identification is becoming critical to ease the communication between developers in case of global software development or to better know members of large software communities. To quickly identify who are the experts that will best perform a given development task, both the assignment of skills to developers and the computation of their corresponding expertise level have to be automated. Since the real level of expertise is tedious to assess, our challenge is to identify developers having a significant level of experience with respect to a skill. Method: In this paper we propose XTic, an approach that takes up this challenge with the intent to be accurate and efficient. XTIC provides a language to specify skills. It also provides an automatic process that extracts skills and experience levels from source code repositories. Our approach is based on the idea that an expert has a high level of experience with respect to a skill. Results: We have validated XTIC both on open source and industrial projects to measure its accuracy and its efficiency. The results we obtained show that its accuracy is between moderate and strong and that it scales well with medium and large size software projects. Conclusion: XTIC supports the specification of a diversity of developer skills and the extraction of the expertise of these developers under the form of level of experience.
\end{abstract}

\section{INTRODUCTION}

Expert identification is becoming critical to ease the communication between developers in case of global software development $[18,13]$ or to better know members of large software communities $[7,21]$. As software development is more and more complex and requires expertise in many technologies, a particular attention is currently paid to automate the identification of experts with the intent to quickly identify

Permission to make digital or hard copies of all or part of this work for personal or classroom use is granted without fee provided that copies are not made or distributed for profit or commercial advantage and that copies bear this notice and the full citation on the first page. To copy otherwise, to republish, to post on servers or to redistribute to lists, requires prior specific permission and/or a fee.

Copyright 20XX ACM X-XXXXX-XX-X/XX/XX ...\$15.00. who are the best developers to perform a given development task.

From an abstract point of view, expert identification consists in (1) assigning skills to developers but also (2) defining their corresponding expertise level. A skill is an abstract term that can have multiple definitions. It can be very generic such as java, test or web design. It can also be much more precise such as library skills with for example $\mathrm{JUnit}^{1}$ or JQuery $^{2}$. Further, skills can even be specific to a given project or a given team of developers such as mastering the 'core' module of the project for example. The levels of expertise for a given skill are values that must belong to an ordinal scale such as high, middle, low or a five stars level for instance. Their intent is to compare the expertise of developers for a given skill in order to rank them.

Any approach that aims to automate the identification of experts has then to provide a process that automatically assigns skills to developers with their expertise levels. To that extent, both the semantics of skills and expertise levels have to be clearly defined. Moreover, the process has to be accurate as it must identify true experts. It should also be deterministic or at least convergent as it should always assign the same skills and expertise levels to the same developers. Moreover, it has to be efficient with the main objective to identify experts as fast as possible, even in a large community of developers.

Many approaches aim to support expert identification. Some of them target specific skills such as code ownership [14] or bug fixing [1]. Other aim to provide an abstract data model that can be queried to ease the identification of experts $[18$, $2]$. To the best of our knowledge, none of them propose to explicitly define the semantics of skills and assign expertise levels, which is the main purpose of our approach. Our purpose is to support project managers who want to assign tasks to their developers, or developers who search collaborators to help them. Our approach takes up the three following challenges:

- How to specify skills?

- How to extract developer's skills and expertise levels?

- How to classify developers to better identify experts?

\footnotetext{
${ }^{1}$ JUnit is a Java library used to develop Unit Tests in Java

${ }^{2}$ JQuery is a JavaScript library that eases many manipulations of the DOM tree
} 
Our approach provides a DSL (Domain Specific Language) for defining what is a skill. Based on this language it provides an extraction mechanism that browses software repositories (currently only source code repositories) to automatically assign expertise levels to developers. The concept of this mechanism is based on the Thomas the Apostle principle : we only rely on the observation of the developers actions to evaluate them. Therefore, instead of computing the expertise level which is subjective and hard to define, we compute the experience level which is objective. Our key hypothesis is that a high level of experience with respect to a skill equals to a high level of expertise for the skill. We then provide an aggregation formulae to rank developers and, on top of it, a simple mechanism to ease the identification of experts.

Our approach is fully implemented in an open source tool, named XTIC. We have validated XTIC by realizing two experiments. First, we identify experts in a sub-part of GitHub that is composed of 16 projects and 280 developers with the intent to stress-test our approach. Our experiment shows that expert identification can be realized for these projects in less than 3 hours. Second, we have used our approach in an industrial case study with the intent to validate the accuracy of our approach. It shows that all the skills that interest our industrial partner can be defined with our language. Further, the experts identified by our approach strongly correspond to the experts manually identified by our industrial partner.

Our paper is organized as follows. Section 2 starts by presenting the related work. Section 3 clarifies the field of expert identification by giving some rigorous definitions. It then deeply presents our approach. Section 4 presents the two validations we have done. Finally Xection 5 discusses the limits of our approach and suggests some improvements and Section 6 concludes.

\section{RELATED WORK}

Expert finding is a prolific topic in the domain of information retrieval $[10,15]$ and is an important subject of the Enterprise tracks of the Text REtrieval Conferences (TREC) since 2005 [4]. However these research approaches are based on generic text analyses and thus cannot fully exploit the data present in a source code repository (history, ownership).

Another example of early work is the Expertise Browser (ExB) [18] proposed by Mockus and Hersleb. They define the concept of experience atom (EA), a basic unit of experience, that can be built from each delta of each files from the VCS of the project. An EA may then be associated with one or multiple domains (functional area, technology used, purpose or type of the change). Finally to measure the level of expertise in a particular domain they count the number of EA associated with this domain. This work is close to our mainstream idea, however there is information on how the set of domains is built and how the domains are specified, and finally how the experience atoms are extracted. We argue that our DSL allows more freedom and flexibility in the specification of the targeted skills.

Specific research approaches targeting the search of experts in the case of a software development project has been accomplished. The Expertise Recommender (ER) [16] proposed by McDonald and Ackerman was one of the first. Their tool relies on two heuristics to identify experts. The first one called change history considers that the revision authors are the experts for the corresponding file. The second one called tech support uses a support database to identify users that already solved problems.

More recently, several research tools [5, 17, 22] were developed to help a developer who is looking for an expert on a file she is modifying or using. They use the touches made on the selected files to determine the list of experts.

As we have seen, some projects rely solely on the analysis of the source code [18] and others attempt to improve the recommendations by looking at other forms of data. For example Moraes et al. with the Conscius tool [19] look at mailing lists.

Codebook [2] is a tool that builds a graph by mining the source code repository, the work item database, the employee directory as well as documents. It also offers a regular expressions language that can be used to search the graph. Based on this framework they have developed several applications over the years. For example, Hoozizat [2], a web search portal for finding the people who own and are responsible for a feature, an API, a product or a service. Another example of application is CARES [13] which is a Visual Studio extension that exposes developers profiles who have contributed to a given file.

Schuler et al. have defined a technique to identify experts of Java methods by looking at the history of usage of a particular method [20]. They argue that developers that use a method should be considered with the same level of expertise than developers that change and edit the method. In their work the unit of experience considered is thus a Java method.

At that point, it is crucial to understand that the domains of expertise we target are not necessarily related to source code areas. For instance, a skill can be defined as the usage of third-party library in the software, which is often scattered in many places of the software. Also, if one want to track who adds or removes the Deprecated annotation on Java elements, we clearly see this is not linked with source code areas. One purpose of our approach is to allow to track either skills related to the implementation of a unit of code (ex: edition of a Java method) or usage of this unit (ex: a call to this method).

Globally our work distinguishes itself from these approaches by providing a way for specifying (with a DSL), extracting (with a tool) and measuring (according to several levels) the developers skills. To our knowledge, no existing work comes up with these three particular features. The DSL not only eases the work of writing down the skills, but is intended to offer much more possibilities when specifiying the targeted skills.

\section{APPROACH}

As a base hypothesis, we consider that skills can be extracted from syntactical modifications performed by developers on the software artefacts of their projects. For instance, we consider that a developer is a Java expert if and only if she has syntactically edited Java files. The main principle of our approach is then to analyze changes made by developers on software artifacts to extract their skills as well as their levels. It should be noted that we deliberately choose to focus on the analysis of software repositories as they contain most of the activity performed by developers. As a consequence, we support only programming skills, i.e. skills that can be extracted from artifacts that are managed within a source code repository.

As there are many definitions of skills, we define them 
using a domain specific language. This language is used to define what are the syntactical changes that a developer must perform on a software artifact to have a corresponding skill. For instance, one may consider that a developer must just modify a Java file to be a Java developer. However, another one may consider that such a definition is too permissive as Java files do not only contain Java code but also comments. She may consider that a developer must create a Java class or modify Java methods to be truly considered as a Java developer.

Once skills have been rigorously defined, our approach uses them while browsing source code repositories to compute developers experience levels. An experience level is a quantitative measure that is used to compare the developers, in order to identify experts. Our extraction mechanism is incremental and has been optimized to scale well and to quickly measure developers skills even in large software projects. The comparison of the experience levels can be facilitated by the use of well known clustering algorithms.

This section presents the two main elements of our approach: the skill definition language, the skill and experience levels extraction process.

To better explain our approach, we introduce a simple example of a Java project managed by a software repository. Figure 1 presents the only Java file contained in this project. This file has been modified three times. The first version of the file $(v 0)$ has been created by Alice. Then the version $v 1$ has been committed by Bob. Finally, the version $v 2$ has been committed by Alice again. We assume that the manager of this project is interested in quantifying developers experience with regard to the JUnit test framework. We assume that she wants to identify two kinds of JUnit expert. The first ones (Test creators) are developers that create tests and the second ones (Test modifiers) are the developers that update existing tests.

\subsection{Definitions}

To ease the explanation of the approach we first lay out several definitions. Let $D$ be the set of developers, $S$ the set of skills and $L$ the set of experience levels. The goal of any approach that aims to identify experts is to assign skills to developers with a corresponding experience level. In other word, such an approach computes a binding function that builds triples $(d, s, l) \in D \times S \times L$. Our approach computes such a binding function by analyzing software repositories. It analyzes all the syntactical changes that are made by developers. To that extent, we define the concept of an atomic file change that is the set of all the modifications performed to a file by a developer during a commit. More formally an atomic file change $c$ is completely defined by the two versions of the file before $\left(f_{0}\right)$ and after $\left(f_{1}\right)$ the commit $\left(c=\left(f_{0}, f_{1}\right)\right)$. The kind of the modification is either defined by the software repository or can be inferred by looking at the two files. If $f_{0}$ does not exist, that means that the file has been created. If $f_{1}$ does not exist, that means that the file has been deleted. If both files exist and have the same name, it means that the content of the file has been modified. If the names of the two files are not the same, it means that the file has been renamed or moved.

Finally, as a commit may target several files and as developers commit several times in a software project, we consider that each developer $d$ performs a set of changes $C_{d}$. The set of changes of each developer can be computed by browsing any software repository. For instance, the repository of Figure 1 contains the two following sets of changes $\left(C_{a}\right.$ for Alice and $C_{b}$ for Bob):

$$
\begin{array}{r}
C_{a}=\left\{\left(\emptyset, \text { Foo.java }_{v 0}\right),\left(\text { Foo.java }_{v 1}, \text { Foo.java }_{v 2}\right)\right\} \\
C_{b}=\left\{\left(\text { Foo.java }_{v 0}, \text { Foo.java }_{v 1}\right)\right\}
\end{array}
$$

Our approach then inputs the set of changes $C_{d}$ of all developers $d$ and performs an analysis to assign skills to developers with a corresponding experience level. This analysis consists in matching changes $\left(c \in C_{d}\right)$ against syntactical patterns, which have been defined using our domain specific language.

\subsection{Skills definitions by syntactical patterns}

Rather than presenting the syntax of our domain specific language for defining skills, we present in this section its main concepts. We propose to specify a skill as a set of syntactical patterns applying to atomic file changes. If the modifications that a developer performs during an atomic file change match at least one pattern of a skill, then the skill is assigned to the developer or his experience regarding this skill is increased.

A skill definition may contain several patterns. Moreover, a developer may perform several atomic file changes. As a consequence, several matches occur for one developer and one skill during the analysis of a software repository. The number of matches corresponds to the level of the skill. A level then corresponds to the number of editing operations performed by a developer that match a skill definition.

A syntactical pattern of a skill defines a syntactical modification that can be done on the file target of an atomic file change. The modification can be done on the file (create the file, delete it or change its name) or can be done on its content (change a line or a token). We then defines a syntactical pattern as a chain of four filters: a path filter, a kind filter, a content filter and a tree modification filter. These four filters are chained for the sake of efficiency. All atomic file changes that have been filtered out by one filter will not be checked by the next filters.

The kind filter applies first to the kind of change that is made to the file target of the atomic change file. Then, the path filter constraints the path of either the source or target file, or both. This last case can be useful do detect moved files during refactoring for instance. The content filter and tree modification filter apply to the content of the file. The content filter considers that the content is a sequence of character while the tree modification filter considers that the content is an abstract syntax tree (AST). These two filters can also be computed on the source or target file of the atomic change file.

Kind filter. The purpose of the kind filter is to describe which are the kinds of modification that must be performed by a developer to have the skill. The kinds of modification can be: creation, modification, deletion or renaming. The kind filter is therefore defined by a mask that specifies the allowed kinds of modification. By default, only the creation and the modification of files are allowed. In our example, the JUnit creator can create, modify or rename files whereas the JUnit modifier must only modify or rename them (and not delete or 
Listing 3: Modification of a test method

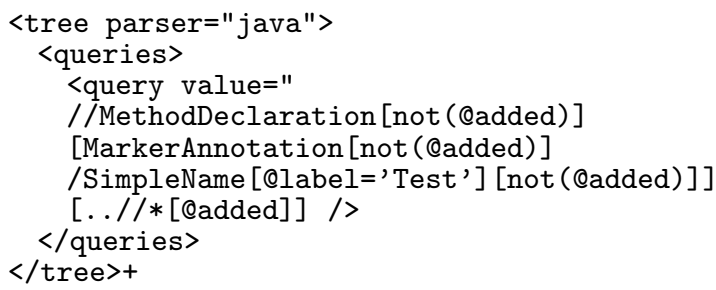

Listing 4: Complete example using the 4 filters proposed by XTiC

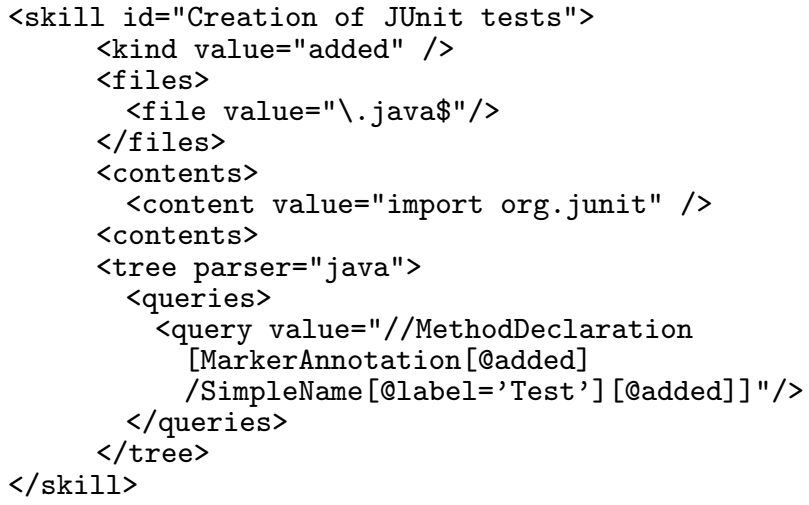

\subsection{Skills and experience levels extraction}

Our skills extraction process is a two steps process. The first step consists in creating the required data model by browsing a software repository. During this first step, a particular attention has to be paid on renamed and moved files as they can have a significant wrong impact on the results of our approach. A renamed/moved file in most VCS (Versioning Configuration System) is seen as a file deletion and creation, performed in the same commit. Therefore, when applying our skills extraction process, all the syntactical patterns contained in the file might be assigned to the developer that performed the renaming/moving, even if she performed no modification at all on the file content. To avoid that, we introduce a move/renaming detection algorithm that we run when extracting a set of changes from a VCS. This algorithm focuses on commits where there are both deleted $D$ and added files $A$. On these commits, we compute the normalized compression distance of each element in $D \times A$. Whenever this distance is below a threshold 0.25 , and whenever it involves two files that have no better distance with another file, we mark the files has being moved/renamed.

The second step aims at assigning skills to developers and to compute a level, which is a positive integer that represents how many times a syntactical pattern has been matched. This step is quite straightforward. It iterates on the atomic file changes of each developer. For each atomic file change, all the patterns that define a skill definitions are checked. As previously explained, skill definitions are composed of several syntactical patterns. These patterns are checked iteratively against changes and return each one a positive integer. These integers are finally aggregated into one integer that corresponds to the level of the skill. By default they are aggregated using a sum, but it is possible to provide a custom formula.

To simplify the analysis of the results of the skill extractor, we propose to apply a post-processing step. Its purpose is to map the levels of skills into a smaller ordinal scale such as low, medium and high. We propose to use the K-means clustering algorithm [8] to map the developers into the clusters. This algorithm creates clusters of values regarding their distance. Configured to output three clusters, it then groups lower values together, as well as higher values and medium values. Thanks to this step, one can then ask for an expert with a high level. This post-processing is optional as it depends on the number of developers being analyzed. If this number is too small, the clusters will not reflect the developer skills. In this case, the integer value is more meaningful.

Conceptually, our approach allows to analyze several software repositories with a same set of skills. However, we currently do not provide a module for merging developer profiles and experience levels scattered on several projects. However, this aspect has already been studied and should be easily included in XTIC in a future work [12].

\section{VALIDATION}

In this section we first describe the implementation of XTic. We then stress test our approach on several opensource projects, to evaluate its performance. Finally, we evaluate the accuracy of our approach in a case study conducted with AKKA, our industrial partner.

Our validation then aims to answer to the following research questions:

- Is XTic able to analyze a large-scale set of data? (RQ1)

- Are the results computed by XTIC correct? (RQ2)

\subsection{Implementation}

XTIC has been written in Java on top of the Harmony [9] framework. Harmony is an infrastructure designed to ease the development of tools that mine software repositories. It provides an abstract model that can be used to specify analysis of commits. Thanks to this model, HARMONY analyses such as XTIC can be run on many version control systems such as Git, SVN, Mercurial, TFS or CVS. The XTIC analysis also relies on the Eclipse JDT parser to perform the parsing of the Java files. The building of the clusters of developers is done by using the implementation of the K-means clustering provided by Weka [6].

\subsection{Stress test}

The purpose of this stress test is to answer the first research question (RQ1): is XTIC able to analyze a large-scale set of data? Since this analysis depends on the syntactical patterns, the size of the source code and the number of versions, it is not possible to make a single experiment to provide a definitive answer. We thus choose to apply some worst case scenarios to a corpus of 16 Java projects. All these project are hosted on GiTHuB, they are active and have more than one developer. They vary from 140 commits with 2,097 lines of code ( $\mathrm{LoC}$ ) for nanohttpd to 1,419 commits with 79,236 Java LoC (crunch) ${ }^{3}$. We argue that the 16 projects

${ }^{3}$ More details on the corpus may be found at http://se. labri.fr/xtic/ 

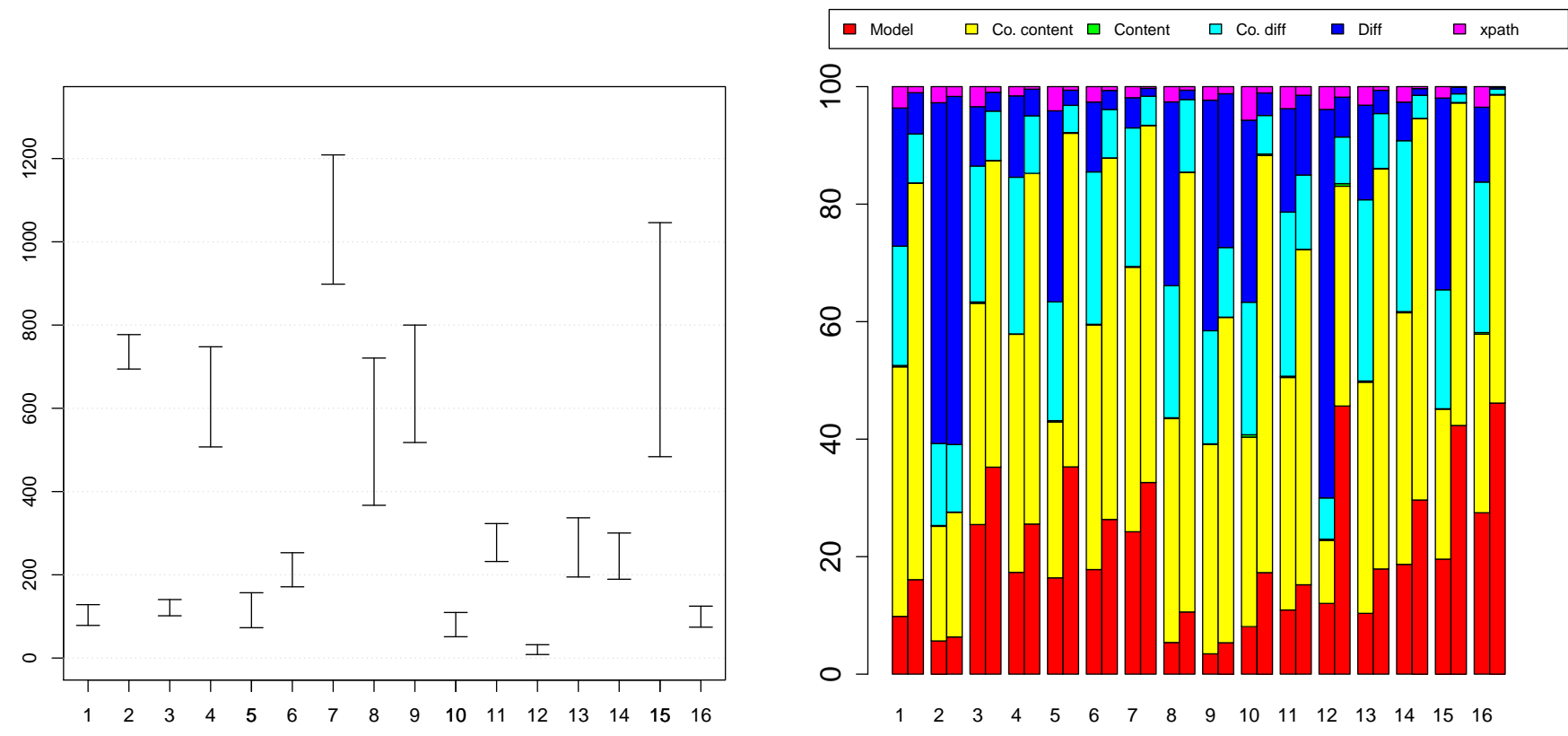

(a) Time by project in seconds (high: worst case, low: JUnit). (b) Breakdown \% of running time (left: worst case, right: JUnit). Figure 2: Time and breakdown of XTIC worst case versus JUnit test on a Java corpus.

selected for this experiment will provide a solid insight of the performance of XTIC in action.

As described in Section 3, a syntactical pattern may contain filters on file names, kinds of modification (creation, edition), file content and tree modifications. Since the two first filters are almost free to check, this experiment focuses on the last two. Searching for file content requires reading files, thus the worst case is to search for an absent string in all files. Since we are unable to figure out what is the worst case of the tree modification filter, i.e., an XPath query, we define three queries on Java files which have an heavy load:

- all nodes added: the XPath engine has to return plenty of results.

- new local variable: this requires to find specific nodes and most commits introduce variables.

- genericity usage: since genericity may be found on class declaration or instantiation this query has to perform disjunctions.

This worst case scenario is compared with another one that only includes the JUnit skill definition. This definition considers that a JUnit tester is a developer that performs modifications on Java files that contain the string org. junit and where a method annotated by a JUnit has been introduced or changed. It corresponds to the JUnit creator and modifier patterns described in Section 3.

Experiments are performed on a Intel Core i7 Cpu M640 at $2.80 \mathrm{Ghz}$ with $8 \mathrm{Go}$ of RAM running with Ubuntu 13.04 (kernel 3.8.0-24) and OpenJDK 1.7.0_25. All these scenario (each part of the worst case and JUnit) are run many times (more than 5) on different passes to avoid IO cache effects and only the worst times are reported. The Figure 2 is twofold, the left-hand side shows the whole computation time (y-axis) for each project (x-axis) as segments. The upper end of each segment represents the worst case scenario, while the lower end represents the JUnit scenario. The right-hand side of Figure 2 shows a breakdown of these running times as percentages. Each group of two bars represents a single project, where the left bar shows the worst case scenario and the right one the JUnit scenario. Breaks represents respectively (bottom up) the time spent analyzing the VCS (Model), the time of checking out the files in order to search for patterns (Co. content), the time of searching effectively these patterns (Content), the time for checking out the previous version of a file (Co. diff) in order to build ASTs and doing the tree differentiation (Diff), and finally the time consumed by the XPath queries (xpath).

\section{Conclusion.}

This experiment shows mainly two things. First XTIC analysis is generally fast enough, even on large scale project, which answer our RQ1. On average, the worst case scenario (resp. JUnit scenario) is under 7 minutes (resp. 5 minutes) with a maximum of 20 minutes (resp. 15 minutes) for a project containing 1,282 revision (with $135,644 \mathrm{LoC}$ in the last version) - it is worth noting that it is not even the biggest project neither from the number of commits nor the number of LoC.

Moreover this experiment shows some of the design choice of XTIC. When looking only the left bars, i.e., the worst case scenario, the time is mostly spent in doing tree differentiation which makes sense since all possible diffs are computed. On the more realistic example of JUnit (right bars) a simple content filter avoids most of them, thus the biggest part of 
time is spent doing the checkout of files. In any case the matching inside the files itself is almost free compared to other things (when carefully looking at the graphs it can be barely seen). As a first advice learned from this experiment, if possible, any skill definition should contain a content filter to avoid wasting time in tree differentiation. Adding more XPath queries is not expensive compared to the whole process as soon as a diff is already computed. Computing many skills in a single pass should be preferred in order to avoid redoing tree differentiation.

When looking at the breakdowns, the project 2 seems really singular since both bars look like the same. After manually inspecting this project, we found that $70 \%$ of the files contains test code. Again even though JUnit rules are more realistic, the whole computation time is thus subsumed by tree differentiation which explains this result.

\subsection{Industrial case study}

The purpose of this case study is to answer the second research question (RQ2): are the results computed by XTIC correct?. To that extent we perform an experiment on an industrial project where we compare the results of XTIC with developers expertise estimated by a manager and a software architect.

\subsubsection{Setup}

We first present the software project analyzed in this case study, as well as the skills required by our industrial partner.

Project and developers. Our industrial partner AKKA Technologies ${ }^{4}$ agreed to grant us access into a software project repository and to provide us a list of developers skills they are interested in. The project studied is a document management system which development started in 2009 and contains 3 years of development activity. A total of 13 developers are involved in this project. The software is mainly written in Java and contains 53 Java KLOC at its latest version.

Skills definitions. We asked to our industrial partner to define the set of developers skills they wanted to measure. A meeting of 2 hours with 2 project managers was necessary to come up with a set of 8 high-level skills that encompass 28 concrete skills according to our definiion. They were described in natural language first. The 2 hours include the time to introduce XTIC capabilities to the 2 project managers. Then, we wrote the skill definitions to integrate them into XTic. We were able to express all the skills in about 15 minutes. It turned out that each skill necessitate several patterns, and only file and content filters were necessary to write these patterns, as shown in Table 1. XTic was therefore expressive enough to fit the requirements of our industrial partner. The skills definitions are not freely available for the sake of confidentiality.

Developers skills. We asked our industrial partner to deliver several skills evaluations indicating for each developer $d \in P_{D}$ and each skill $s \in S$ (as shown in Table 1), what is the expertise level $l \in L=\{$ low, medium, high $\}$ of the developer w.r.t. the skill. We end up therefore with several ternary relations that are subsets of $P_{D} \times S \times L$. The project manager and architect accepted to complete an independent

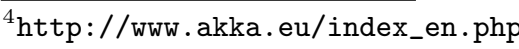

Table 1: Number of patterns and filters in the skill definitions.

\begin{tabular}{rccccc}
\hline & & \multicolumn{4}{c}{ \#Filters } \\
\cline { 3 - 6 } Skill & \#Patterns & File & Kind & Content & Tree \\
\hline config & 5 & 5 & 0 & 6 & 0 \\
workflow & 2 & 2 & 0 & 2 & 0 \\
domain & 2 & 2 & 0 & 5 & 0 \\
modeling & 7 & 7 & 0 & 0 & 0 \\
graphics & 3 & 3 & 0 & 4 & 0 \\
jsf & 2 & 2 & 0 & 1 & 0 \\
plugins & 4 & 4 & 0 & 1 & 0 \\
tests & 3 & 3 & 0 & 3 & 0 \\
\hline Total & 28 & 28 & 0 & 22 & 0 \\
\hline
\end{tabular}

Table 2: Distribution of the developers in the levels for the evaluations $E_{\text {ar }}$ and $E_{\mathrm{ma}}$.

\begin{tabular}{|c|c|c|c|c|c|c|}
\hline \multirow[b]{2}{*}{ Skill } & \multicolumn{2}{|c|}{ \#Low } & \multicolumn{2}{|c|}{ \#Medium } & \multicolumn{2}{|c|}{ \#High } \\
\hline & $E_{\text {ar }}$ & $E_{\mathrm{ma}}$ & $E_{\text {ar }}$ & $E_{\mathrm{ma}}$ & $E_{\text {ar }}$ & $E_{\mathrm{ma}}$ \\
\hline config & 7 & 7 & 2 & 3 & 4 & 3 \\
\hline workflow & 7 & 9 & 2 & 2 & 4 & 2 \\
\hline domain & 7 & 7 & 4 & 0 & 2 & 6 \\
\hline modeling & 6 & 11 & 5 & 0 & 2 & 2 \\
\hline graphics & 4 & 5 & 0 & 7 & 9 & 1 \\
\hline jsf & 3 & 3 & 4 & 2 & 6 & 8 \\
\hline plugins & 9 & 10 & 0 & 0 & 4 & 3 \\
\hline tests & 3 & 5 & 4 & 1 & 37 & 7 \\
\hline total & 46 & 57 & 21 & 15 & 37 & 32 \\
\hline
\end{tabular}

evaluation based on their personal knowledge. We therefore have two evaluations $E_{\text {ar }}$ and $E_{\mathrm{ma}}$, which distribution in the levels are shown in Table 2. As these two persons disagree on several developers, we have also built another relation $E_{\text {ag }}$ computed from $E_{\text {ar }}$ and $E_{\text {ma }}$, where we kept only the triples $(d, s, l)$ that were included both in $E_{\text {ar }}$ and $E_{\mathrm{ma}}$. This relation contains thus only the developers and skills on which both persons agreed. Its distribution in the levels is shown in Table 3.

The evaluations $E_{\mathrm{ar}}$ and $E_{\mathrm{ma}}$ contain $13 * 8=104$ triples. The evaluation $E_{\text {ag }}$ contains 48 triples. It means that the two persons agreed only on about one developer out of two. Table 2 indicates that the proportion of low, medium and high developers is similar globally but can vary a lot in the rules (in graphics for instance). Low developers are the most common, followed closely by high developers. Medium developers are less frequent. Table 3 indicates that the two persons agree more frequently on the high and low developers than on medium developers. This is probably due to the fact that it is easier to know if a developer is high or low, but the border between low and medium on one hand, and medium and high on the other hand, is fuzzy and very subjective.

\subsubsection{Experiment}

We ran XTIC on the software project repository using the previously described skills definitions to compute an integer score for each of the 13 developers on each of the 8 skills. To convert the integer into a level, we used the K-means clustering algorithm configured to produce three clusters, as explained in Section 3. It led to an evaluation called $E_{\mathrm{xt}}$. To be able to compare XTIC on the evaluation $E_{\mathrm{ag}}$, that contains only the developers for which the manager and architect agreed, we computed an evaluation $E_{\mathrm{xt}^{\prime}}$ containing 
Table 3: Distribution of the developers in the levels for the evaluation $E_{\text {ag. }}$.

\begin{tabular}{rccc}
\hline Skill & \#Low & \#Medium & \#High \\
\hline config & 4 & 1 & 2 \\
workflow & 6 & 1 & 1 \\
domain & 4 & 0 & 1 \\
modeling & 5 & 0 & 0 \\
graphics & 0 & 1 & 0 \\
jsf & 2 & 0 & 3 \\
plugins & 8 & 0 & 2 \\
tests & 2 & 0 & 5 \\
\hline total & 31 & 3 & 14 \\
\hline
\end{tabular}

Table 4: Agreements (\#A), disagreements (\#D), strong disagreements (\#SD) and Kappa between XTIC $\left(E_{\mathrm{xt}}\right)$, the manager $\left(E_{\mathrm{ma}}\right)$ and the architect $\left(E_{\mathrm{ac}}\right)$.

\begin{tabular}{ccccc}
\hline Couple & \#A & \#D & \#SD & Kappa \\
\hline$\left\{E_{\mathrm{ma}}, E_{\mathrm{ac}}\right\}$ & 48 & 56 & 21 & 0.26 \\
$\left\{E_{\mathrm{xt}}, E_{\mathrm{ma}}\right\}$ & 67 & 37 & 6 & 0.59 \\
$\left\{E_{\mathrm{xt}}, E_{\mathrm{ac}}\right\}$ & 59 & 45 & 14 & 0.42 \\
\hline$\left\{E_{\mathrm{xt}^{\prime}}, E_{\mathrm{ag}}\right\}$ & 41 & 7 & 1 & 0.85 \\
\hline
\end{tabular}

only the developers and skills contained in $E_{\mathrm{ag}}$.

\subsubsection{Results}

To measure the agreements among the three evaluations, we used Cohen's kappa coefficient [3]. This coefficient is a value between 0 and 1 , with 1 meaning a perfect agreement and 0 no agreement. The comparison of XTIC evaluation $\left(E_{\mathrm{xt}}\right)$ with the one of the manager $\left(E_{\mathrm{ma}}\right)$, the one of the architect $\left(E_{\mathrm{ac}}\right)$, and between these two persons are exposed in Table 4. In this table, strong disagreement means that a person assigned a low level while the other assigned high level. A detailed comparison is also shown in the Venn diagram from Figure 3.

First of all, the agreement between the architect and the manager is poor (Kappa of 0.26). Moreover they have many strong disagreements (about 20\%). This shows that evaluating developers skills is complex and prone to subjectivity. Moreover, it means that XTIC results can not conform with both the manager and the architect. The agreement between XTIC and both the manager and architect is fair (Kappa of resp. 0.59 and 0.42 ), which is a satisfying point. Moreover there are fewer strong disagreements for XTIC with both the manager and architect. The agreement with the manager $(0.59)$ is good, and the number of strong disagreement is very low (only about 5\%). The results therefore indicate that $\mathrm{XTIC}$ have a similar opinion to the manager.

Since the architect and manager significantly disagree, we produced an evaluation $E_{\text {ag }}$ where we kept only the triples $(\mathrm{d}, \mathrm{s}, \mathrm{l})$ where the manager and architect assigned the same skill level. The developers and skills contained in this evaluation have therefore a good confidence. This evaluation contains 48 triples, which is still a fair number of developers and skills. As shown in Table 4, the results of XTIC on the developers and skills of $E_{\text {ag }}$ contains 41 agreements and only 7 disagreements leading to a Kappa coefficient of 0.85 that indicates an almost perfect agreement.

To have a more precise view of the results of XTIC, we computed the precision and recall of XTIC with regard to

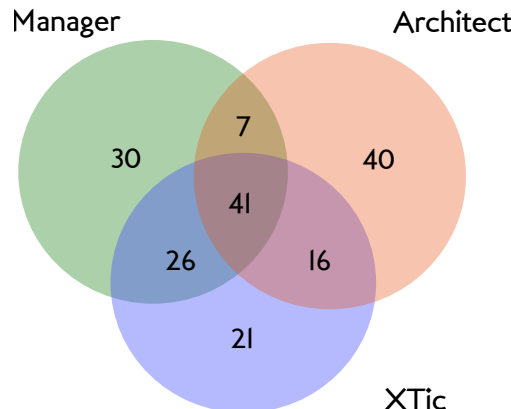

Figure 3: Agreement distribution of the results. Our approach has 21 times disagreed with both the manager and the architect.

Table 5: Precision and recall of XTIC for each level of the $E_{\text {ag }}$ relation.

\begin{tabular}{|c|c|c|c|c|c|}
\hline \multicolumn{2}{|c|}{ Low } & \multicolumn{2}{|c|}{ Medium } & \multicolumn{2}{|c|}{ High } \\
\hline $\begin{array}{c}\text { Prec. } \\
0.94\end{array}$ & $\begin{array}{c}\text { Rec. } \\
0.94\end{array}$ & $\begin{array}{c}\text { Prec. } \\
0.29\end{array}$ & $\begin{array}{c}\text { Rec. } \\
0.67\end{array}$ & $\begin{array}{c}\text { Prec. } \\
1.0\end{array}$ & $\begin{array}{c}\text { Rec. } \\
0.71\end{array}$ \\
\hline
\end{tabular}

each level of skill, as shown in Table 5. We can see that the recall and precision for the low and high levels range from good to perfect. On the contrary, the precision on the medium class is poor, while the recall is just fair. This confirms that a human is more prone to assign either a low or high level, while a medium evaluation is more likely to be given by XTIC. A medium level remains fuzzy from a human point of view.

\subsubsection{Discussion}

Since a manual analysis of each case of disagreement would have taken too much time, we presented to our industrial partner only the strong disagreements of the manager, the architect and XTIC during a meeting where both the architect and the manager were present. They were asked to reach an agreement on each case. With regard to the cases of strong disagreements between the architect and the manager, it turned out that in every case, the manager was right. The reason is because the manager is closer to the developers and therefore knows better the work they do every day and the skill they have. On the contrary, the architect assign tasks to the developers, but does not ensure that they perform it in person. Therefore they have a biased vision of the developers skills. However, a possible threat to this experiment is the lower hierarchical position of the architect compared to the project manager whose ascendency may have prevailed over the architect point of views.

Also, with regard to XTIC, only six cases remain of strong disagreements unexplained with the manager. After analyzing these cases, we understood that the manager was right, but he confirmed that these particular skills were not used on the project we analyzed, the developers being assigned to other coding tasks.

This conclusion was very interesting for our industrial partner since knowing whom of the architects and the managers has a better vision of the developers skills has led to an internal debate. Unfortunately, the lack of time granted by our partner prevented us to question directly the developers on their opinion on each others. We are conscious that team members are fairly good at evaluating at each others. 
Finally, we observed that only the content filter and path flter were used. We argue that the usage proportion of each type of filter is variable, and there exists cases where the kind filter and tree filter are needed, like shown earlier in the description of the language.

\section{LIMITS AND IMPROVEMENTS}

The previous section shows that XTIC has a good accuracy and a good efficiency. While it offers some new supports to the field of expert identification, it still suffers from some limits. In this section we highlight these limits and explain how we can handle them.

First of all, XTIC is based on a syntactical analysis of source code. The diff technique it uses currently does not support refactoring operations such as method renames or moves. These operations are therefore considered to be delete and add operations. They have then a strong impact on the skill and experience level extraction as a developer that just renames or moves a method will be considered to have all the skills that are related to the content of the method. To overcome this limitation, we think about using more efficient diff techniques such as Change Distilling for instance [11]. Furthermore, the parser used by XTIC currently cannot handle files that contain more than one programming language since they are complex to parse. Such files are for instance HTML files that contain both HTML and JavaScript code. To handle them, we need to define specific parsers and diff techniques.

Secondly, XTIC support expert identification by analyzing all the commits performed by developers on a software repository. Therefore it does not reflect the real expertise level of a developer but rather her experience level. As a consequence, XTIC will not identify as experts developers that do not make a lot of work. For instance, newcomers will always be identified as non expert even if they do have a strong knowledge. This limit comes from the base hypothesis of XTIC that states that an expert is a developer who committed in her field of expertise. However, we can overcome it by performing analysis according to a time window (some weeks or some months) with the objective to normalize production of newcomers with the one of older developers. Similarly, a high level of experience possibly does not necessarily reflect that the developer produces high quality work with respect to the skill. If such quality can be expressed using XTIC, then we will be able to distinguish developers that produce such high quality work.

In this paper, the DSL proposed by XTIC was designed to uniformly specify a large range of skills. In practice, XTIC is intended to help project managers and developers who are willing to track a defined set of skills. However, this early paper does not give any clue on how much this DSL can be appropriated by a person who does not know XTIC. Indeed, in the experiments we wrote the skills, and we rather focused on the results that are produced. In a future work, we will have to evaluate how people appropriate our DSL so that they can use it without our intervention. But more generally, we claim that the usage of XTIC definitively requires a good knowledge of the technical aspects of the software to be analyzed in order to efficiently benefit the tool.

Finally, the language proposed by XTIC to define skills can be improved. Currently it does not allow for conjunction of skill patterns. However, we think that there exists skills that are spread over more than one file per commit. For instance, in a Java context we can define a library update skill, which consists in modifying the JUnit JAR file and in the same time editing the test files adequately. Moreover even though the expressiveness of our language remains tedious to validate, we observed that we managed to specify a various range of skills. In that direction, additional requirements and feedbacks from both industrial partners and open source communities would help us to improve our language, and better understand their needs.

\section{CONCLUSION}

The identification of expert is one of the current challenges of software engineering. In this paper we propose to take up this challenge by analyzing source code changes that are managed by a software repository.

Our approach, named XTIC, proposes a domain specific language to specify skills. It then automatically extracts developers experience by browsing software repositories and by matching skills definitions to the work committed by the developers. Finally, it provides some simple support to rank developers and hence to ease the identification of expert.

Our approach is based on the hypothesis that a developer has to commit work in her field of expertise to be considered as an expert. Our approach is then more like Thomas the Apostle as it needs to observe commits and does not rely on any other claim.

We have validated our approach by stress testing its implementation to show its efficiency and by checking its accuracy. Our validation shows that it is quite efficient and that its accuracy is between moderate to strong. Without being a silver bullet, XTIC provides a support for those who want to identify experts in an automated manner.

As a further work, we first think about overcoming some of the limits of XTIC. In particular, we are working on improving the parsers we rely on and on adding time windows in the extracting process. We also think about integrating XTIC with other approaches that are also based on syntactical analysis but that target software artefacts other than source code, such as mails or bug reports for instance. Also, our filter-based approach only targets atomic file changes included in commits on software repositories. A perspective is to extend this language so that it can apply on higher level of granularities, such as a commit, i.e. a set of atomic file changes. It would allow to observe developers that perform tasks on two different files for instance. We could go even further by defining patterns that apply on a project history, i.e. a set of commits.

\section{REFERENCES}

[1] J. Anvik, L. Hiew, and G. C. Murphy. Who should fix this bug? In Proceedings of the 28th international conference on Software engineering, ICSE '06, page 361-370, New York, NY, USA, 2006. ACM.

[2] A. Begel, K. Y. Phang, and T. Zimmermann. Codebook: Discovering and Exploiting Relationships in Software Repositories. 2010.

[3] J. Cohen. A coefficient of agreement for nominal scales. Educational and Psychological Measurement, 20(1):37-46, Apr. 1960.

[4] N. Craswell, I. Soboroff, and A. P. Vries. Overview of the TREC 2005 enterprise track. 2005.

[5] D. Cubranic, G. Murphy, J. Singer, and K. Booth. Hipikat: a project memory for software development. 
Software Engineering, IEEE Transactions on, 31(6):446-465, 2005.

[6] R. De War and D. Neal. WEKA machine learning project: Cow culling. Technical report, The University of Waikato, Computer Science Department, Hamilton, New Zealand, 1994.

[7] G. Demartini. Finding experts using wikipedia. In Proceedings of the Workshop on Finding Experts on the Web with Semantics (FEWS2007) at ISWC/ASWC2007, Busan, South Korea, page 33-41, 2007.

[8] R. O. Duda, P. E. Hart, and D. G. Stork. Pattern Classification (2nd Edition). Wiley-Interscience, 2000.

[9] J.-R. Falleri, C. Teyton, M. Foucault, M. Palyart, F. Morandat, and X. Blanc. The harmony platform. Technical report, Univ. Bordeaux, LaBRI, UMR 5800, Sept. 2013.

[10] H. Fang and C. Zhai. Probabilistic models for expert finding. In Proceedings of the 29th European conference on IR research, ECIR'07, page 418-430, Berlin, Heidelberg, 2007. Springer-Verlag.

[11] B. Fluri, M. Würsch, M. Pinzger, and H. Gall. Change distilling: Tree differencing for fine-grained source code change extraction. IEEE Trans. Software Eng., 33(11):725-743, 2007.

[12] M. Goeminne and T. Mens. A comparison of identity merge algorithms for software repositories. Science of Computer Programming, (0):-, 2011.

[13] A. Guzzi and A. Begel. Facilitating communication between engineers with CARES. In Proceedings of the 2012 International Conference on Software Engineering, ICSE 2012, page 1367-1370, Piscataway, NJ, USA, 2012. IEEE Press.

[14] H. Kagdi, M. Hammad, and J. Maletic. Who can help me with this source code change? In Software Maintenance, 2008. ICSM 2008. IEEE International Conference on, pages 157-166, 2008.

[15] C. Macdonald, D. Hannah, and I. Ounis. High quality expertise evidence for expert search. In Proceedings of the IR research, 30th European conference on Advances in information retrieval, ECIR'08, page 283-295, Berlin, Heidelberg, 2008. Springer-Verlag.

[16] D. W. McDonald and M. S. Ackerman. Expertise recommender: a flexible recommendation system and architecture. In Proceedings of the 2000 ACM conference on Computer supported cooperative work, CSCW '00, page 231-240, New York, NY, USA, 2000. ACM.

[17] S. Minto and G. Murphy. Recommending emergent teams. In Fourth International Workshop on Mining Software Repositories, 200\%. ICSE Workshops MSR '07, pages 5-5, 2007.

[18] A. Mockus and J. D. Herbsleb. Expertise browser: A quantitative approach to identifying expertise. In In proceedings of International Conference on Software Engineering (ICSE 2002, page 503-512, 2002.

[19] A. Moraes, E. Silva, C. da Trindade, Y. Barbosa, and S. Meira. Recommending experts using communication history. In Proceedings of the 2nd International Workshop on Recommendation Systems for Software Engineering, RSSE '10, page 41-45, New York, NY, USA, 2010. ACM.

[20] D. Schuler and T. Zimmermann. Mining usage expertise from version archives. In Proceedings of the 2008 international working conference on Mining software repositories, MSR '08, page 121-124, New York, NY, USA, 2008. ACM.

[21] R. Sindhgatta. Identifying domain expertise of developers from source code. In Proceedings of the 14 th ACM SIGKDD international conference on Knowledge discovery and data mining, KDD '08, page 981-989, New York, NY, USA, 2008. ACM.

[22] Y. Ye, K. Nakakoji, and Y. Yamamoto. Reducing the cost of communication and coordination in distributed software development. In B. Meyer and M. Joseph, editors, Software Engineering Approaches for Offshore and Outsourced Development, number 4716 in Lecture Notes in Computer Science, pages 152-169. Springer Berlin Heidelberg, Jan. 2007. 